\title{
Wearable piezoresistive strain sensor based on graphene-coated three-dimensional micro-porous PDMS sponge
}

\author{
Young Jung ${ }^{1,2}$, Kyungkuk Jung ${ }^{2,3}$, Byunggeon Park ${ }^{1,2}$, Jaehyuk Choi ${ }^{2}$, Donghwan Kim², Jinhyoung Park ${ }^{4}$, \\ Jongsoo $\mathrm{Ko}^{2}$ and Hanchul $\mathrm{Cho}^{1 *}$ (])
}

\begin{abstract}
We present a highly elastic and wearable piezoresistive strain sensor based on three-dimensional, micro-porous graphene-coated polydimethylsiloxane (PDMS) sponge suitable for being attached on human skin. The proposed strain sensors are simply fabricated by a sugar templating process and dip coating method based graphene ink in a facile and cost effective manner. The fabricated graphene-coated PDMS sponge shows highly stable mechanical properties in various tensile stress-strain test. A graphene thin film coated onto the backbone of PDMS sponges is used as the sensing materials of piezoresisitve strain sensors. The changes in resistance of the devices are highly stable, repeatable, and reversible when various strain is applied. Furthermore, the strain sensors show excellent sensing performance under different strain rate and mechanically robustness enough to be worked stably under repeated loads without any degradation.
\end{abstract}

Keywords: Graphene, PDMS, Strain sensor, Porous sponge

\section{Introduction}

Recently, there has been attracting attention in order to develop wearable sensors that can be applied to variety of applications including electronic skin [1, 2], soft robotics [3-5], health monitoring [6, 7], and human-machine interfaces [8]. For these fields of wearable applications, it is greatly important that to make various key factors such as low fabrication cost, sensitivity, long-term stability, and highly conformable properties with high performance. Conventionally, strain gauge based on metal foil have been widely used to measure deformation or strain on such objects. Even though fabrication process of conventional strain gauges is well-established technology such as traditional micro electro mechanical systems (MEMS), they have poor stretchability because they use rigid materials $[9,10]$. Due to the rigidity and small

\footnotetext{
*Correspondence: hc.cho@kitech.re.kr

1 Precision Machining Control Group, Korea Institute of Industrial Technology, 42-7, Baegyang-daero 804beon-gil, Sasang-gu, Busan 46938, Republic of Korea

Full list of author information is available at the end of the article
}

working range, the strain gauges are unable to detect large strain in wearable applications.

To overcome these fundamental limitations of metal strain gauges, research efforts have focused on widening the detection range of strain sensors. The proposed method for the fabrication of sensors is to use of nanomaterials such as Carbon Nanotubes (CNTs), Carbon Blacks (CBs), Graphene, Zinc Oxide Nanowires ( $\mathrm{ZnO}$ NWs), Siliver Nanowires (AgNWs) that can be coupled with elastic polymer. These nanomaterials based strain sensors is a promising materials of choice due to their excellent mechanical and electrical properties [11-14]. In particular, piezoresistive strain sensors can be manufactured with simple method using elastic polymer with conducting nanomaterials [3, 14-18]. Amjadi et al. [11] suggested a strain sensor with tunable gauge factor (GF) in the range of 2 to 14 based on connection-disconnection of AgNW network in elastic polymer. Lee et al. [19] fabricated a wearable strain sensors for human motion detection based on silver nanoparticle (Ag NP) thin film to change the electrical resistance caused by the opening/ 
closure of micro-cracks. Fan et al. [20] reported a highly stretchable strain sensors based on CNTs layer on a thermoplastic polyurethane (TPU) multifilament, especially the strain to failure of $1500 \%$. As such, studies using nanomaterials with elastic polymer, which transduce applied strain into an electrical resistance, have been widely used due to the ease of signal processing. However, the sensors manufactured in the form of thin film suffered from low GF under 10, high hysteresis due to the viscoelastic properties of elastic polymer and slip-page of nanomaterials under stretching/releasing cycles.

Studies on porous sponge have focused on enhancing the sensitivity of the sensors with reduced hysteresis properties. The polymer-based porous sponge, which can be manufactured through various methods such as sugar templating process [14, 21, 22], gas-forming [23, 24], and 3-D printing [25-27], has the advantage of being easily deformed even at a small force and rapidly recovering. The porous sponge undergoes reversible mechanical deformation due to the reduced viscoelastic properties of polymer and anti-barreling phenomenon [14]. Many studies have been proposed to coat or synthesize nanomaterials on porous sponges and use them as sensing materials of strain sensors based on piezoresistivity. Samad et al. [28] proposed a graphene foam with excellent conductivity, reaching $160 \mathrm{~S} \mathrm{~m}^{-1}$ and high strain-sensing sensor using dip coating and pyrolysis. Li et al. [29] also suggested highly flexible and three-dimensional graphene foam, which is capable of $30 \%$ of its original length, via self-assembly and dry freezing. However, manufacturing of the three-dimensional graphene sponges for strain sensor requires expensive and complicated temperature control such as pyrolysis or low freezing. Despite the high cost fabrication method, there are limitations in stretchability and reliability due to the low bond force between polymer and graphene. Therefore, it is still a great challenge to study to fabricate a strain sensor with easy of fabrication, high sensing performance, as well as excellent reliability during repeated the stretching/releasing cycles.

In this work, we report a highly elastic and wearable strain sensor utilizing three-dimensional, micro-porous graphene-coated polydimethylsiloxane (PDMS) sponges that are capable of measuring strains. The strain sensor based on graphene-coated PDMS sponge, which is highly conductive, flexible, and stretchable, is fabricated by using sugar templating process and dip coating directly onto a bare PDMS sponge without any complicated process. We measured the mechanical properties of porous sponges including tensile stress-strain curves under different strain range and repeated cycles. To verify the sensing performance of the strain sensor, we checked the change in resistance of the sensor under various conditions. Our sensor shows an excellent performance of gauge factor as 19.2 at $50 \%$ strain, high repeatability, and reliability up to 1000 cycles at $20 \%$ strain without any signal degradation.

\section{Fabrication of graphene-coated PDMS sponge}

Figure 1 shows the fabrication process of the graphenecoated PDMS sponge using sugar templating process and dip coating. Firstly, sugar and water are mixed in a weight ratio of 30 to 1 and put in a pre-fabricated flexible mold and becomes a stacked sugar. The stacked sugar is heated for $30 \mathrm{~min}$ at $70{ }^{\circ} \mathrm{C}$ to completely evaporate the water (Fig. 1a) and changes to the cube sugar. A PDMS precursor (Sylgard 184, Dow Corning Corp., USA) with a mixture of resin and hardener in a weight ratio of 10 to 1 is placed in a vacuum chamber (Jeio Tech, Korea) under $1 \times 10^{-1}$ torr for $10 \mathrm{~min}$ to remove the bubbles. The fabricated cube sugar placed onto a Petri dish, and a PDMS was poured into the Petri dish. The PDMS precursor fills the empty space in the cube sugar through capillary force. The cube sugar filled with the PDMS precursor is placed in a thermal oven and cured for $3 \mathrm{~h}$ at $90{ }^{\circ} \mathrm{C}$. After the thermal curing process, the sugar grains were completely dissolved in deionized water and the water inside the PDMS sponge is fully dried (Fig. 1b). The dried PDMS sponge was alternately dipped with $10 \mathrm{~mm} \mathrm{~min}^{-1}$ into graphene ink (MExplorer Co., Ltd, Korea). The graphene-coated PDMS sponges are placed in thermal oven and cured for $30 \mathrm{~min}$ at $100{ }^{\circ} \mathrm{C}$ (Fig. 1c) to dry the ink and increase the adhesion force between the sponge surface and graphene ink. The graphene ink consists of dispersion of graphene in ethanol, dimethylformamide (DMF), and $\mathrm{N}$-methyl-2-pyrrolidone (NMP), in particular, evaporation of ethanol in graphene dispersed ink results in a PDMS sponge with graphene attached on the backbone through strongly physical interaction, due to the $\mathrm{CH}-\pi$ interactions between the methyl groups of PDMS and the $\pi$-surface of graphene [22]. Figure $1 d-i$ show the images of the surface of sugar, bare PDMS sponge, and graphene-coated PDMS sponge via field emission scanning electron microscope. Sugar grains stick together by evaporation of water (Fig. 1d, g). As shown in Fig. 1e, $\mathrm{h}$, the PDMS sponge has a three-dimensional, interconnected, and micro-porous structure and the surface is clean. Figure 1f, i are SEM images of the surface of graphene-coated PDMS sponge, it is seen that the graphene is uniformly and thinly coated onto the surfaces of the micro-pores and formed a networks.

\section{Measurement setup}

The surface of the bare and graphene-coated PDMS sponges is characterized by FE-SEM (S-4800, HITACHI, Japan). Contact angle measurement was performed using 


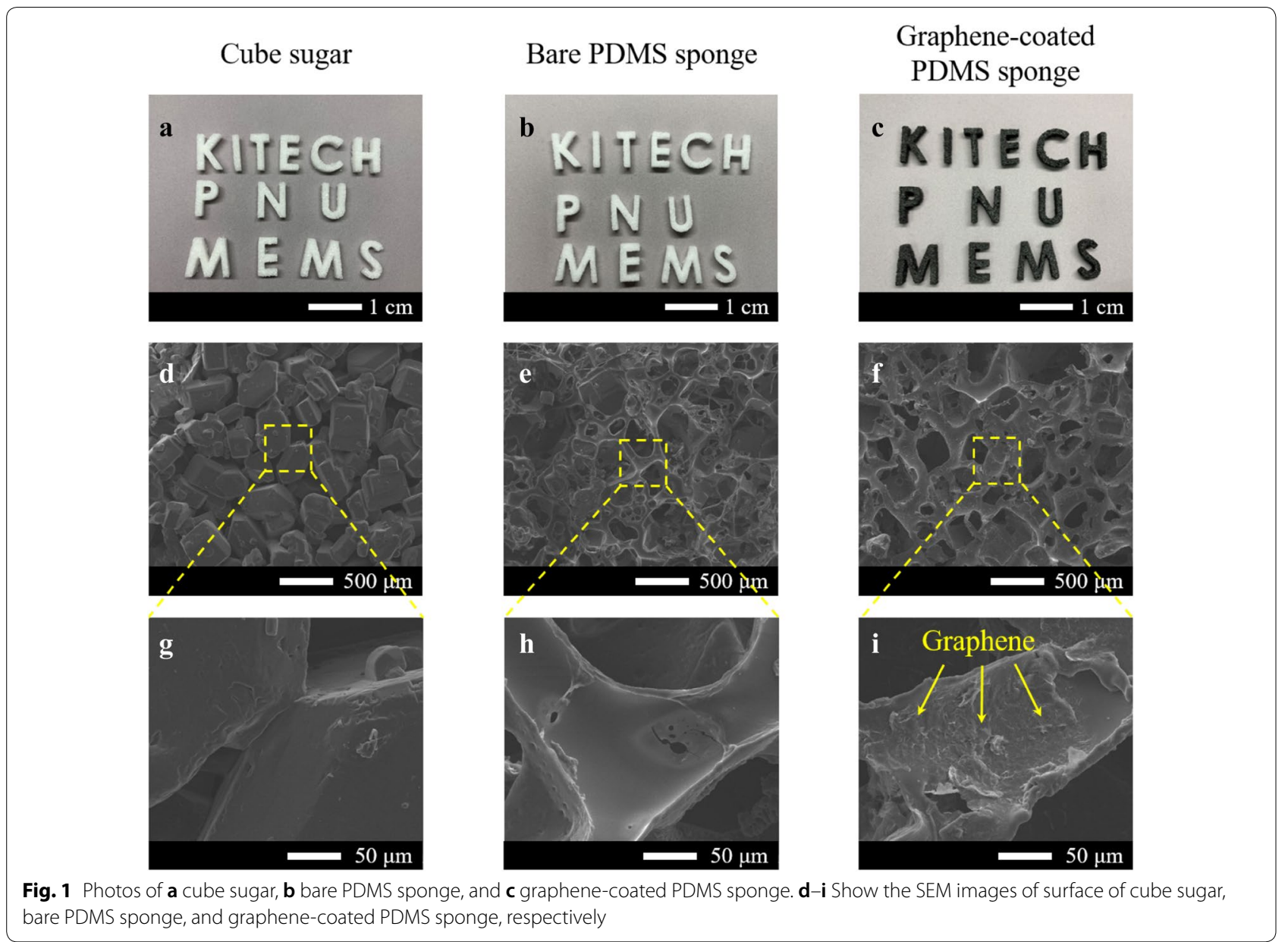

a contact angle analyzer (Phoenix 300 Touch, Surface Electro Optics, Korea) and a DI water droplet of $4 \mu \mathrm{L}$. For the measurement of tensile stress-strain curves of sponges, as shown in Fig. 2a, universal testing machine (JSV-H1000, JISC, Japan) was employed to evaluate the performance with load cell (HF-1, maximum load $10 \mathrm{~N}$, load resolution $0.001 \mathrm{~N}$ ). The sponges were stretched at a speed of $10 \%$ strain $\mathrm{min}^{-1}$ except strain rate test in mechanical evaluation. The test specimens for mechanical properties and strain sensors were prepared at $\mathbf{a}$

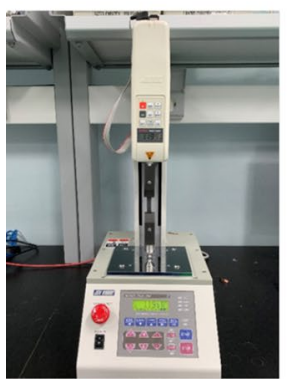

b

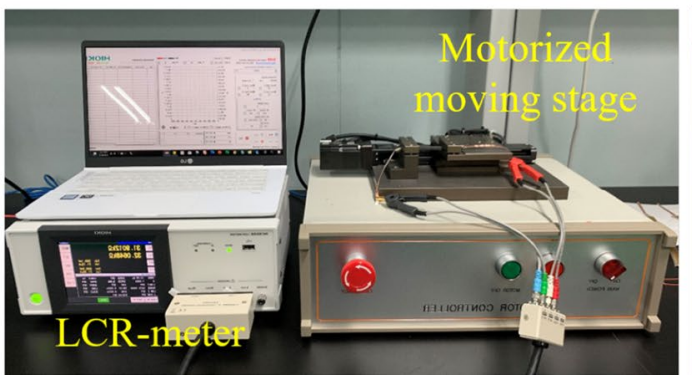

Top view

c (motorized moving stage)

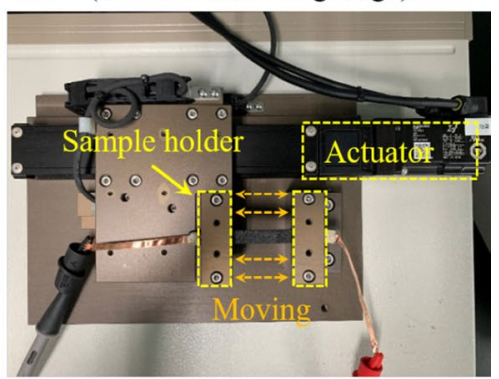

Fig. 2 Experimental setup for the measurement of mechanical properties and changes in resistance for applied strain. a Universal testing machine for measurements of tensile stress-strain. $\mathbf{b}$ Setup of measuring the changes in resistance. $\mathbf{c}$ Image of fabricated horizontally motorized moving stage 
$40 \mathrm{~mm}$ (length, $\mathrm{l}) \times 10 \mathrm{~mm}$ (width, $\mathrm{w}) \times 3 \mathrm{~mm}$ (thickness, $\mathrm{t})$ and $100 \mathrm{~mm}(\mathrm{l}) \times 10 \mathrm{~mm}(\mathrm{w}) \times 3 \mathrm{~mm}(\mathrm{t})$, respectively. An LCR-meter (HIOKI-3536, HIOKI, Japan) was used to measure the DC resistance of the sensors. All sensor evaluations were measured by connecting LCR-meter and computer in real time (Fig. 2b). We manufactured a tensile tester using a linear axis encoder (SRSH24YN-200, Misumi, Japan) and a linear actuator (LX2001CP, Misumi, Japan), controlled via Labview program (Fig. 2b, c). The voltage versus current characteristics were measured using a Parameter analyzer (Keithley 4200 SCS, Tektronix, USA). The copper tapes, which were the electrodes, were attached on two opposite sides of graphene wrapped sponge using silver conductive epoxy (CW-2400, ITW Chemtronics, USA).

\section{Results and discussion}

The surface properties of bare and graphene-coated PDMS sponge was studied by contact angle measurement. Figure 3 shows the contact angle measurement results for the bare and graphene-coated PDMS sponges. The measured contact angle was the average obtained from five measurement results from each sponges at different points. The measured water contact angle was $108 \pm 1.7^{\circ}$ on the surface of the bare PDMS sponge, and $131 \pm 2.5^{\circ}$ on the surface of the graphene-coated PDMS sponge. PDMS is basically hydrophobic property with a water contact angle of $100^{\circ}$, which is further enhanced by a porous sponge that reduces the contact area between droplet and solid surface $[30,31]$. Coating with graphene further increases the contact angle as $131^{\circ}$ for the enhanced roughened surface due to nanostructured by of graphene [22]. The low standard deviation as 2.5 of measurement results also shows that the graphene ink is fully and uniformly coated on the surface of PDMS sponge.

The sponge is highly elastic and flexible, and it can be deformed with variation shapes without plastic deformation. To quantitatively evaluate the mechanical properties of bare and graphene-coated PDMS sponges, we measured the tensile stress-strain as a function of maximum strain range, strain range, and repeated cycles. Figure 4a shows an evaluation images when graphenecoated PDMS sponge is stretched from 0 to $60 \%$. Figure $4 \mathrm{~b}$ indicates the tensile stress-strain curves of two sponges until the failure. In cases of bare PDMS sponge, maximum tensile stress of about $72.9 \mathrm{kPa}$ was required for $77.7 \%$ strain, while graphene-coated PDMS sponge required a pressure of $80.9 \mathrm{kPa}$ for the $68 \%$ strain. The larger pressure required for the same deformation after graphene coating is due to the increased stiffness of the sponge with coating of graphene that is excellent mechanical properties. Although the tensile strain has been reduced due to the increased stiffness, it can be deformed up to $60 \%$ tensile range, making it suitable for a variety of wearable systems such as human motion detection [32]. Furthermore, we measured the tensile stressstrain under various strain (Fig. 4c). In can be seen that the maximum stress increases with increasing strain, and the curve is divided into two distinct stages. Figure 4d indicates the cycles test results. A 20\% strain was applied to the strain sensors with 1000 cycles repeatedly, the permanent deformation occurred at the strain within $1 \%$, but the tensile stress is almost similar.

The graphene-coated PDMS sponge can be used for wearable applications such as strain sensors because its conductivity, flexibility, and stability. When the sponge is stretched, graphene network changes along with the opening/closure of micro-pores, which causes the change of the conductive network. The sensing performance of the strain sensors based on graphene-coated PDMS sponge was measured under static loads. Figure $5 \mathrm{a}$ depicts the relative resistance variation of the strain sensor in a $40 \%$ strain range. The resistance variations were not significant up to $10 \%$ strain range, but it a

Bare PDMS sponge

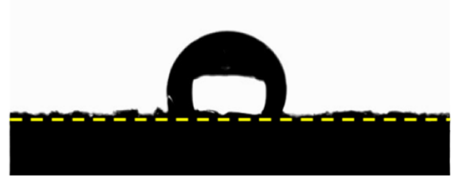

b Graphene-coated
PDMS sponge

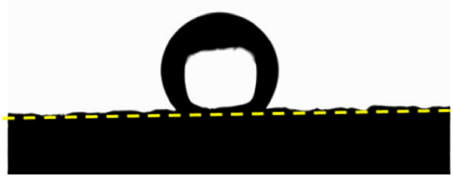

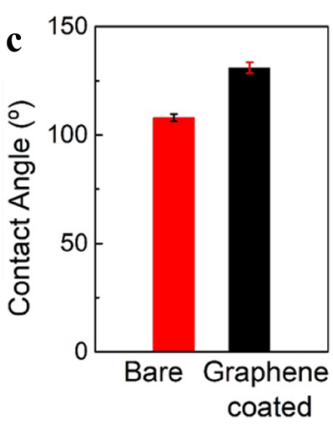

Fig. 3 Static water contact measurements performed on $\mathbf{a}$ bare and $\mathbf{b}$ graphene-coated PDMS sponge. $\mathbf{c}$ The water contact angle was $108 \pm 1.7^{\circ}$, and $131 \pm 2.5^{\circ}$ on the surface of bare and graphene-coated PDMS sponge, respectively. The water contact angle was increased by the hydrophobic property of the PDMS and the enhanced roughened surface due to the porous structure and nanostructure 


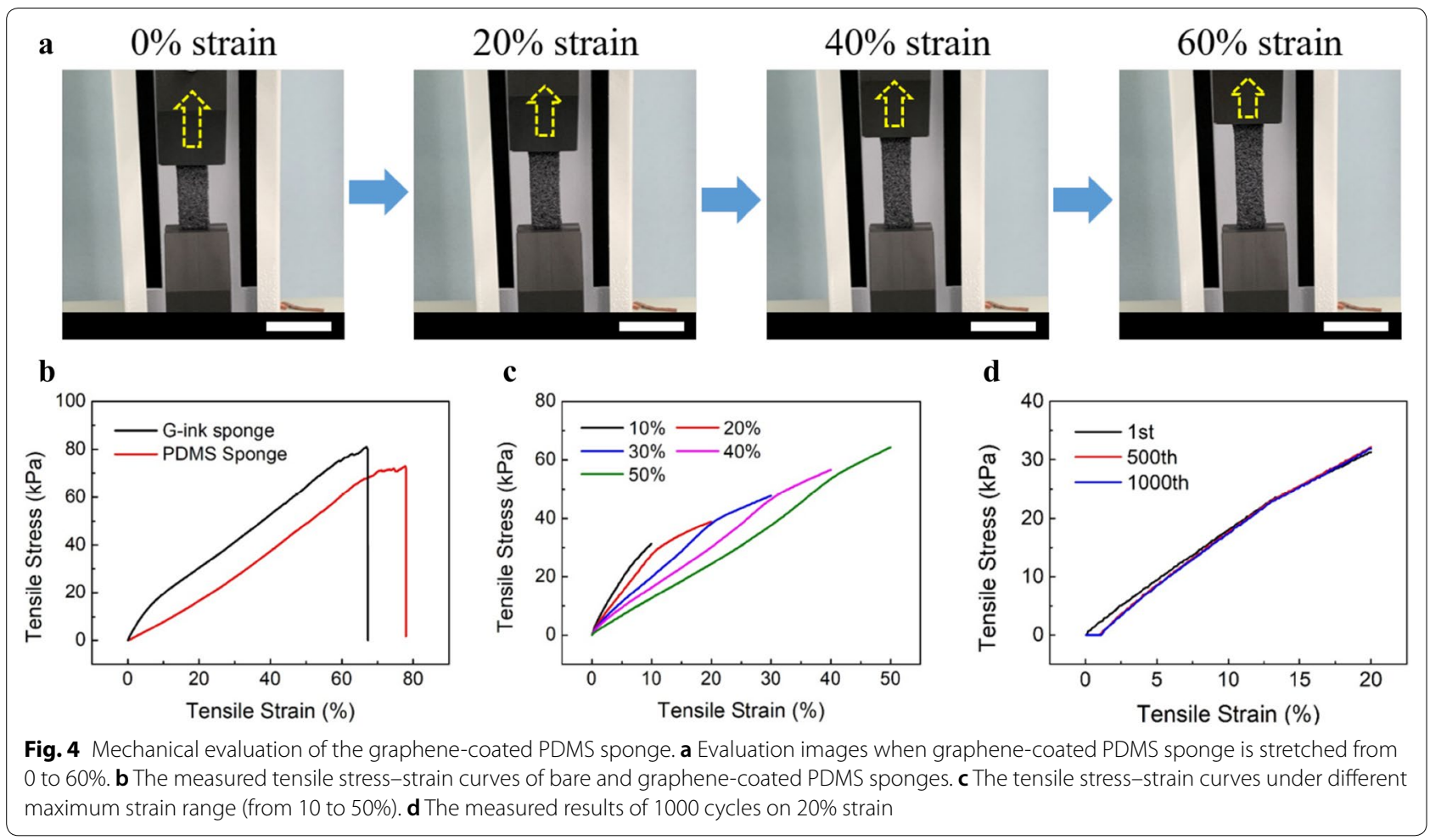

was confirmed that the increase rapidly in the $10-30 \%$ range. The stretching and releasing curves is almost overlap, sensors showed negligible hysteresis and this has been attributed to the reversible deformation of opening/closure of micro-pores. Figure $5 \mathrm{~b}$ shows the voltage $(V)$ versus current $(I)$ characteristics of graphene-coated PDMS sponge at different applied strains. It is obviously observed the slope of the $\mathrm{V}$ versus I curves decreases with the applied strains. The slope on the V versus I curves is the resistance by Ohm's law, it means that the electrical resistance is dramatically changed by the applied strain. Figure $5 \mathrm{c}$ presents the relative resistance $\left(\Delta R / R_{0}=\left(R-R_{0}\right) / R_{0}\right.$, where $R$ and $R_{0}$ denote the resistance values with and without applied strain) change over fifteen cycles according to time. The maximum strain is increased from 5 to $50 \%$ with same strain rate $\left(10 \%\right.$ strain $\left.\min ^{-1}\right)$, the minimum strain is fixed at $0 \%$. In $5 \%$ strain, the $\Delta R / R_{0}$ decreases slightly from 0.096 in the first cycle and to 0.089 in the 15 th cycle. However, after $10 \%$ strain, it can be seen that the $\Delta R / R_{0}$ are highly stable in all cycles. When the maximum strain is increased from 10 to $50 \%$, the value of $\Delta R / R_{0}$ also increased by 0.363 to 9.604. Furthermore, there is stable signal in the relationship between the $\Delta R / R_{0}$ according to repeated cycles, indicating excellent reliability of the graphene-coated PDMS sponge in the various strain range. The sensitivity (gauge factor, $\delta\left(\Delta R / R_{0}\right) / \varepsilon$, where $\varepsilon$ denotes the applied strain) to strain at 10 to $50 \%$ was calculated to be 3.63 , $6.43,8.82,13.09$, and 19.21 , respectively. It can be seen that the sensitivity of the strain sensor exponentially increases with increase of the maximum strain. The sensitivity exponentially increases by strain, theses high sensitivity of 19.21 and 50\% strain range sufficient enable the strain sensors based on graphene-coated PDMS sponge to detect a variety of human body motion.

The flexible strain sensors are mainly used to detect deformation of the human body, knee and elbow deform rapidly and repeatedly. Therefore, it is important that the sensing performance have to maintain without any signal degradation in dynamic as well as static loads for real-time applications. Figure 6a shows the measurements of changes in resistance according to strain rate. The strain rate was varied from 0.2 to $0.6 \mathrm{~Hz}$. The average resistance change was 2.558 at $0.2 \mathrm{~Hz}$ and 2.639 and 2.678 at 0.4 and $06 \mathrm{~Hz}$, respectively. Although the resistance increased by $4.6 \%$ as the strain rate changed from 0.2 to $0.6 \mathrm{~Hz}$, this means that the sensing performance is highly stable under diverse strain rates, it can be used to variety of applications. To investigate the durability of strain sensor, 1000 cycles of repeated stretching/releasing with a strain of $10 \%$ applied to the sensor at a $10 \%$ strain $\min ^{-1}$ for $11,000 \mathrm{~s}$. Figure $6 \mathrm{~b}$ indicates the full and enlargements of each 10 cycles measurement results of reliability. The initial, 

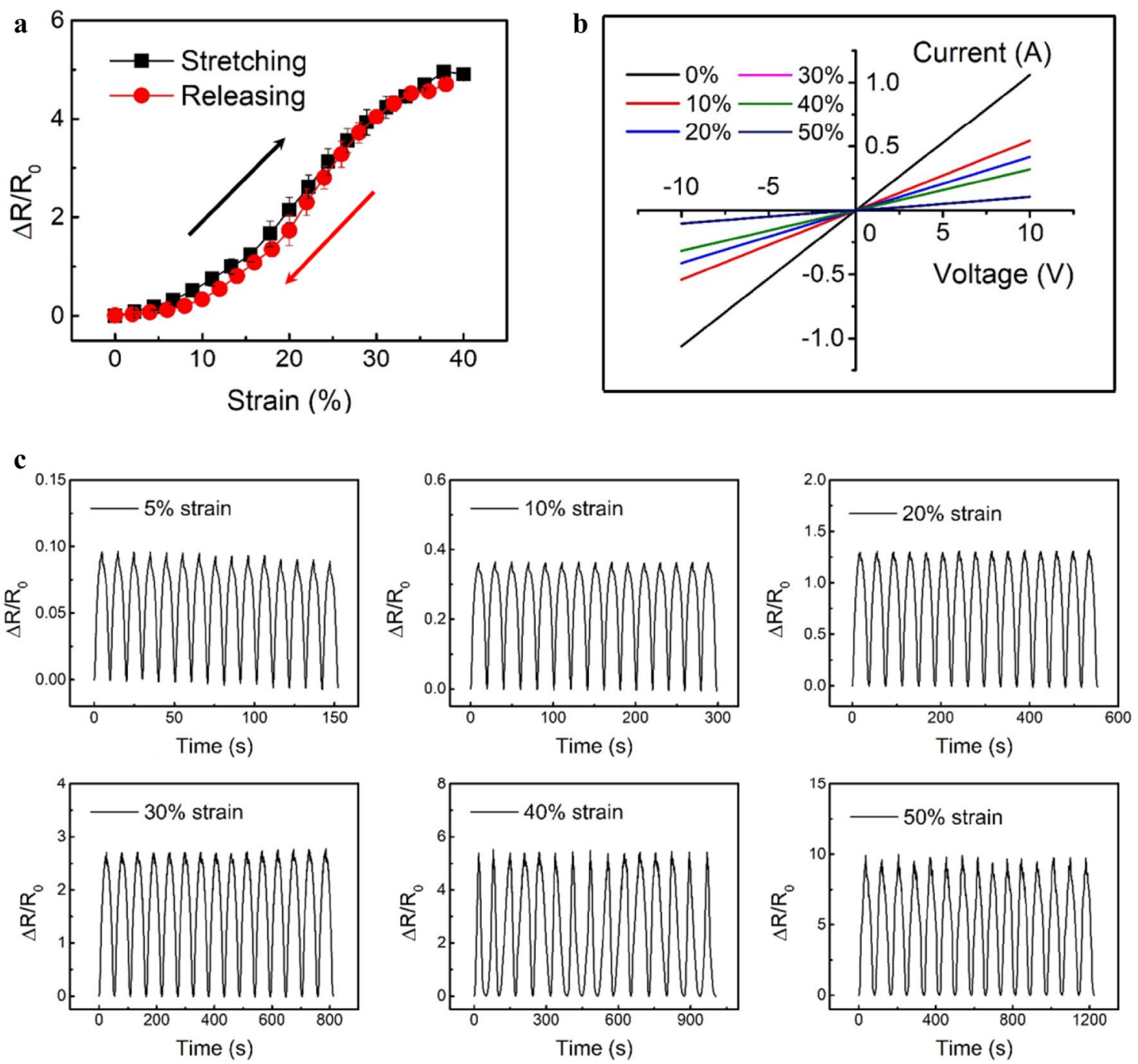

Fig. 5 The measured sensing performance of the piezoresistive strain sensor based on graphene-coated PDMS sponge. a Relative resistance variation of the strain sensor in a $40 \%$ strain with stretching/releasing. b Voltage versus current characteristics of sponge at different applied strain. $\mathbf{c}$ The measured relative resistance change over fifteen cycles according to time with various maximum strain

middle, and last 10 cycles are shown in the red, green, and blue graph, respectively. The measured average resistance values were 1.186 in the initial cycles, 1.178 in the middle cycles, and 1.171 in the last cycles. The decreased change in resistance is only $1.3 \%$, and these values can be negligible for practical applications. The reason why the graphene-coated PDMS sponge shows high stability and reliability is that the graphene bonds strongly to the backbone of PDMS due to the $\mathrm{CH}-\pi$ interactions, and the graphene does not peel-off even after repeated cycles. From these experimental results, we can verify that our strain sensors have good signal stability under static and dynamic loads against repeated stretching/releasing cycles.

\section{Conclusion}

In summary, we have developed a highly elastic and wearable piezoresistive strain sensor based on threedimensional, micro-porous graphene-coated PDMS sponge, which is suitable for being attached on the curvature surface with several excellent properties in terms of the electrical and mechanical performance. The proposed strain sensors were easily produced by a sugar templating process and dip coating method based graphene dispersed ink without any additional process. The fabricated graphene-coated PDMS sponge was able to be deformed up to $60 \%$ strain and showed stable mechanical properties even under various strains and repeated cycles. Because of the changed conductive network between 

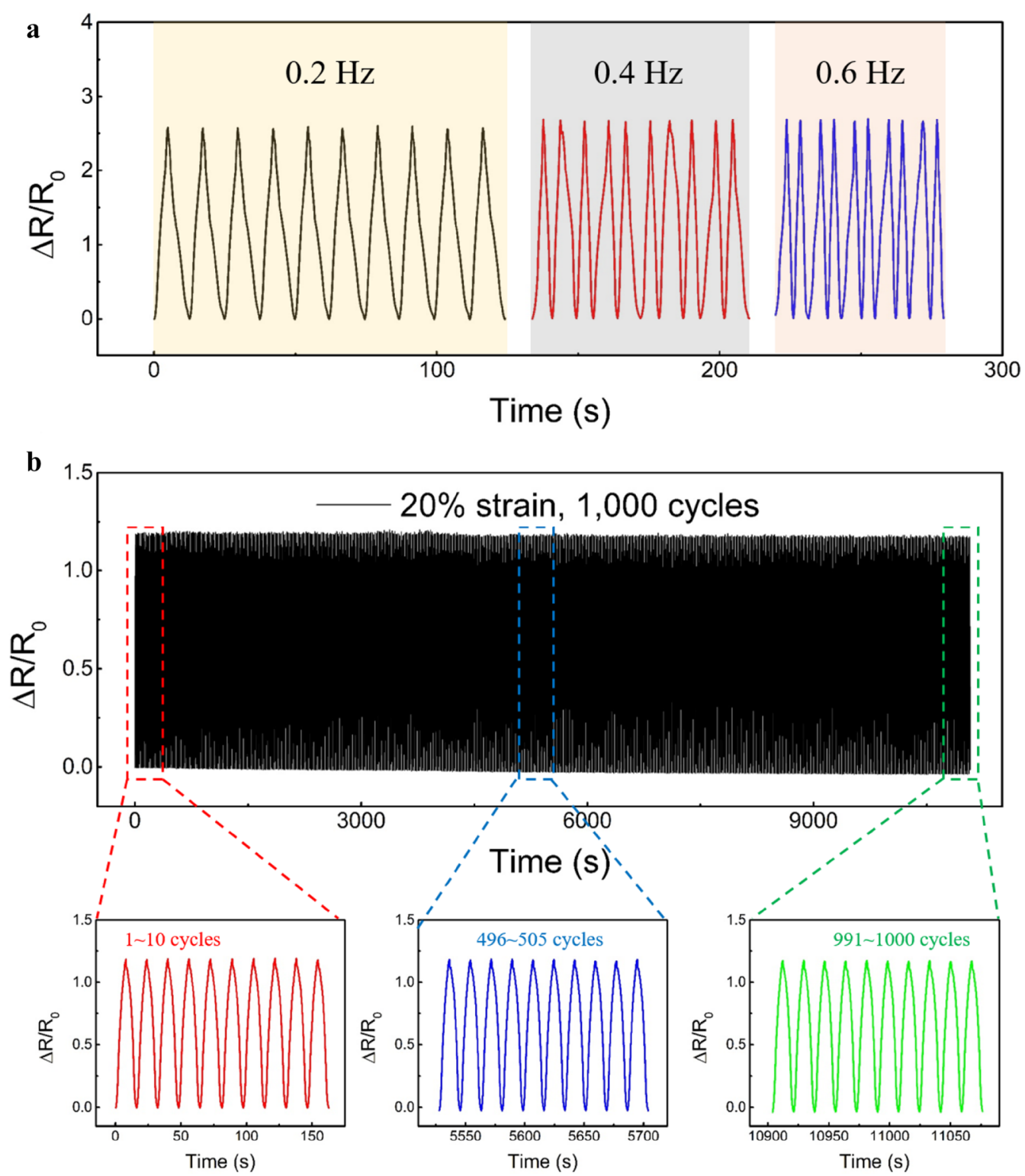

Fig. 6 The measured sensing performance of the piezoresistive strain sensor under dynamic loads. a The measurements of changes in resistance according to strain rate. $\mathbf{b}$ Full and enlargements of each 10 cycles measurement results of reliability

graphene-coated on the micro-pores, the strain sensor showed a high sensitive as 19.21 at $50 \%$ strain, with high repeatability and low hysteresis. Furthermore, we verified the strain sensor had stable sensing performance in various strain rates and high reliability in 1000 times repeated cycles. Finally, we expect our study will contribute toward the development of sensitive and wearable strain sensors that can be applied to various applications including electronic skin, soft robotics, health monitoring, and human-machine interfaces.

\section{Abbreviations}

PDMS: polydimethylsiloxane; MEMS: micro electro mechanical systems; CNTs: carbon nanotubes; CBs: carbon blacks; ZnO NWs: zinc oxide nanowires; AgNWs: silver nanowires; AgNPs: silver nanoparticle; GF: gauge factor; V: voltage; $A$ : current; $R$ : resistance values with applied strain; $R_{0}$ : resistance values without applied strain; $\varepsilon$ : applied strain.

\section{Acknowledgements}

Not applicable.

\section{Authors' contributions}

YJ and HCC developed the idea. KKJ, BGP carried out fabrication and literature search. YJ, JYC, and DHK contributed to measurement. YJ, JHP, and JSK drafted the manuscript. HCC supervised the research and reviewed the manuscript. All authors read and approved the final manuscript. 


\section{Funding}

This study has been conducted with the support of the Korea Institute of Industrial Technology (KITECH) under the "Research Source Technique Project" (EO-19-0009).

\section{Availability of data and materials}

All data generated or analyzed during this study are included in this published article.

\section{Competing interests}

The authors declare that they have no competing interests.

\section{Author details}

${ }^{1}$ Precision Machining Control Group, Korea Institute of Industrial Technology, 42-7, Baegyang-daero 804beon-gil, Sasang-gu, Busan 46938, Republic of Korea. ${ }^{2}$ Graduate School of Mechanical Engineering, Pusan National University, Busandaehak-ro 63beon-gil, Geumjeong-gu, Busan 46241, Republic of Korea. ${ }^{3}$ Atmospheric Environment Research Center, Energy \& Marine Research Division, Korea Marine Equipment Research Institute (KOMERI), Busan, Republic of Korea. ${ }^{4}$ Mechatronics Technology Convergence R\&D Group, Korea Institute of Industrial Technology, 320 Techno sunhwan-ro, yuga-myeon, Dalseong-gun, Daegu 42994, Republic of Korea.

\section{Received: 29 August 2019 Accepted: 14 October 2019}

\section{Published online: 22 October 2019}

\section{References}

1. Wang X, Dong L, Zhang H, Yu R, Pan C, Wang ZL (2015) Recent progress in electronic skin. Adv Sci 2(10):1500169

2. Chortos A, Liu J, Bao Z (2016) Pursuing prosthetic electronic skin. Nat Mater 15:937-950. https://doi.org/10.1038/nmat4671

3. Yan C, Wang J, Kang W, Cui M, Wang X, Foo CY, Chee KJ, Lee PS (2014) Highly stretchable piezoresistive graphene-nanocellulose nanopaper for strain sensors. Adv Mater 26:2022-2027. https://doi.org/10.1002/ adma.201304742

4. Cohen DJ, Mitra D, Peterson K, Maharbiz MM (2012) A highly elastic, capacitive strain gauge based on percolating nanotube networks. Nano Lett 12:1821-1825. https://doi.org/10.1021/nl204052z

5. Tee BC-K, Wang C, Allen R, Bao Z (2012) An electrically and mechanically self-healing composite with pressure- and flexion-sensitive properties for electronic skin applications. Nat Nanotechnol 7:825-832. https://doi. org/10.1038/nnano.2012.192

6. Gong T, Zhang H, Huang W, Mao L, Ke Y, Gao M, Yu B (2018) Highly responsive flexible strain sensor using polystyrene nanoparticle doped reduced graphene oxide for human health monitoring. Carbon 140:286295. https://doi.org/10.1016/j.carbon.2018.09.007

7. Liu C, Choi J (2009) An embedded PDMS nanocomposite strain sensor toward biomedical applications. In: 31 st Annual International Conference of the IEEE EMBS. IEEE, New York, pp 6391-6394

8. Jung S, Kim JH, Kim J, Choi S, Lee J, Park I, Hyeon T, Kim DH (2014) Reverse-micelle-induced porous pressure-sensitive rubber for wearable human-machine interfaces. Adv Mater 26:4825-4830. https://doi. org/10.1002/adma.201401364

9. Huang X, Liu Y, Cheng H, Shin W, Fan JA, Liu Z, Lu C, Kong G, Chen K, Patnaik D, Lee S, Hage-ali S, Huang Y, Rogers JA (2014) Materials and designs for wireless epidermal sensors of hydration and strain. Adv Funct Mater 24:3846-3854. https://doi.org/10.1002/adfm.201303886

10. McEvoy MA, Correll N (2015) Materials that couple sensing, actuation, computation, and communication. Science (80-). https://doi.org/10.1126/ science.1261689

11. Amjadi M, Pichitpajongkit A, Lee S, Ryu S, Park I (2014) Highly stretchable and sensitive strain sensor based on silver-elastomer nanocomposite. ACS Nano 8:5154-5163. https://doi.org/10.1021/nn501204t

12. Yamada T, Hayamizu Y, Yamamoto Y, Yomogida Y, Izadi-Najafabadi A Futaba DN, Hata K (2011) A stretchable carbon nanotube strain sensor for human-motion detection. Nat Nanotechnol 6:296-301. https://doi. org/10.1038/nnano.2011.36

13. Cai L, Song L, Luan P, Zhang Q, Zhang N, Gao Q, Zhao D, Zhang X Tu M, Yang F, Zhou W, Fan Q, Luo J, Zhou W, Ajayan PM, Xie S (2013)
Super-stretchable, transparent carbon nanotube-based capacitive strain sensors for human motion detection. Sci Rep 3:1-9. https://doi. org/10.1038/srep03048

14. Xiao X, Yuan L, Zhong J, Ding T, Liu Y, Cai Z, Rong Y, Han H, Zhou J, Wang ZL (2011) High-strain sensors based on ZnO nanowire/polystyrene hybridized flexible films. Adv Mater 23:5440-5444. https://doi. org/10.1002/adma.201103406

15. Lu N, Lu C, Yang S, Rogers J (2012) Highly sensitive skin-mountable strain gauges based entirely on elastomers. Adv Funct Mater 22:4044-4050. https://doi.org/10.1002/adfm.201200498

16. Shin UH, Jeong DW, Park SM, Kim SH, Lee HW, Kim JM (2014) Highly stretchable conductors and piezocapacitive strain gauges based on simple contact-transfer patterning of carbon nanotube forests. Carbon 80:396-404. https://doi.org/10.1016/j.carbon.2014.08.079

17. Liu C, Choi J (2014) Microelectronic engineering analyzing resistance response of embedded PDMS and carbon nanotubes composite under tensile strain. Microelectron Eng 117:1-7. https://doi.org/10.1016/j. mee.2013.11.013

18. Lee C, Jug L, Meng E, Lee C, Jug L, Meng E (2014) High strain biocompatible polydimethylsiloxane-based conductive graphene and multiwalled carbon nanotube nanocomposite strain sensors High strain biocompatible polydimethylsiloxane-based conductive graphene and multiwalled carbon nanotube nanocomposite strain sensors. Appl Phys Lett. https:// doi.org/10.1063/1.4804580

19. Lee J, Kim S, Lee J, Yang D, Park BC, Ryu S, Park I (2014) A stretchable strain sensor based on a metal nanoparticle thin film for human motion detection. Nanoscale 6:11932-11939. https://doi.org/10.1039/C4NR03295K

20. Fan Q, Qin Z, Gao S, Wu Y, Ma E, Zhu M (2012) The use of a carbon nanotube layer on a polyurethane multifilament substrate for monitoring strains as large as 400\%. Carbon 50:4085-4092. https://doi.org/10.1016/j. carbon.2012.04.056

21. Choi S-J, Kwon T-H, Im H, Moon D-I, Baek DJ, Seol M-L, Duarte JP, Choi Y-K (2011) A polydimethylsiloxane (PDMS) sponge for the selective absorption of oil from water. ACS Appl Mater Interfaces 3:4552-4556. https://doi. org/10.1021/am201352w

22. Iglio R, Mariani S, Robbiano V, Strambini L, Barillaro G (2018) [ASAP] Flexible polydimethylsiloxane foams decorated with multiwalled carbon nanotubes enable unprecedented detection of ultralow strain and pressure coupled with a large working range. ACS Appl Mater Interfaces 10:13877-13885. https://doi.org/10.1021/acsami.8b02322

23. Kobayashi T, Saitoh H, Fujii N, Hoshino Y, Takanashi M (1993) Porous membrane of polydimethylsiloxane by hydrosilylation cure: characteristics of membranes having pores formed by hydrogen foams. J Appl Polym Sci 50:971-979

24. Netti ASODMIA (2009) Design of porous polymeric scaffolds by gas foaming of heterogeneous blends. J Mater Sci Mater Med 20:2043-2051. https ://doi.org/10.1007/s10856-009-3767-4

25. Hinton TJ, Hudson A, Pusch K, Lee A, Feinberg AW (2016) 3D printing PDMS elastomer in a hydrophilic support bath via freeform reversible embedding. ACS Biomater Sci Eng 2:1781-1786. https://doi.org/10.1021/ acsbiomaterials.6b00170

26. Kolesky DB, Homan KA, Skylar-scott MA, Lewis JA (2016) Threedimensional bioprinting of thick vascularized tissues. Proc Natl Acad Sci 113:3179-3184. https://doi.org/10.1073/pnas.1521342113

27. Kolesky DB, Truby RL, Gladman AS, Busbee TA, Homan KA, Lewis JA (2014) $3 \mathrm{D}$ bioprinting of vascularized, heterogeneous cell-laden tissue constructs. Adv Mater 26:3124-3130. https://doi.org/10.1002/adma.20130 5506

28. Samad YA, Li Y, Schiffer A, Alhassan SM, Liao K (2015) Graphene foam developed with a novel two-step technique for low and high strains and pressure-sensing applications. Small 11:2380-2385. https://doi. org/10.1002/smll.201403532

29. Li J, Zhao S, Zeng X, Huang W, Gong Z, Zhang G, Sun R, Wong CP (2016) Highly stretchable and sensitive strain sensor based on facilely prepared three-dimensional graphene foam composite. ACS Appl Mater Interfaces 8:18954-18961. https://doi.org/10.1021/acsami.6b05088

30. Johnson RE Jr., Dettre RH (1963) Contact angle hysteresis. III. Study of an idealized heterogeneous surface. J Phys Chem 107:1744-1750. https:// doi.org/10.1021/j100789a012 
31. Jung Y, Jung KK, Park BG, Ko JS (2018) Capacitive oil detector using hydrophobic and oleophilic PDMS sponge. Int J Precis Eng Manuf Technol 5:303-309. https://doi.org/10.1007/s40684-018-0032-7

32. Amjadi M, Yoon YJ, Park I (2015) Ultra-stretchable and skin-mountable strain sensors using carbon nanotubes-Ecoflex nanocomposites. Nanotechnology. https://doi.org/10.1088/0957-4484/26/37/375501

\section{Publisher's Note}

Springer Nature remains neutral with regard to jurisdictional claims in published maps and institutional affiliations.

\section{Submit your manuscript to a SpringerOpen ${ }^{\odot}$ journal and benefit from:}

- Convenient online submission

- Rigorous peer review

- Open access: articles freely available online

- High visibility within the field

- Retaining the copyright to your article

Submit your next manuscript at $\boldsymbol{\nabla}$ springeropen.com 